\title{
Clinical validation of a virtual environment for normalizing eating patterns in eating disorders
}

\author{
Conxa Perpiñá ${ }^{\mathrm{a}, \mathrm{c}, *}$, María Roncero ${ }^{\mathrm{a}}$, Fernando Fernández-Aranda ${ }^{\mathrm{b}, \mathrm{c}, \mathrm{d}}$, \\ Susana Jiménez-Murcia ${ }^{\mathrm{b}, \mathrm{c}, \mathrm{d}}$, Laura Forcano ${ }^{\mathrm{c}}$, Isabel Sánchez ${ }^{\mathrm{b}}$ \\ ${ }^{a}$ University of Valencia, Valencia, Spain \\ ${ }^{\mathrm{b}}$ Department of Psychiatry, University Hospital of Bellvitge-IDIBELL \\ ${ }^{\mathrm{c} C I B E R}$ Fisiopatología Obesidad y Nutrición (CIBERobn), Instituto Salud Carlos III, Spain \\ ${ }^{\mathrm{d}}$ Clinical Sciences Department, University of Barcelona, Spain
}

\begin{abstract}
The purpose of the present study was to examine the clinical validation of a Virtual Reality Environment (VRE) designed to normalize eating patterns in Eating Disorders (ED). The efficacy of VR in eliciting emotions, sense of presence and reality of the VRE were explored in 22 ED patients and 37 healthy eating individuals. The VRE (non-immersive) consisted of a kitchen room where participants had to eat a virtual pizza. In order to assess the sense of presence and reality produced by the VRE, participants answered seven questions with a Likert scale (0-10) during the experience, and then filled out the Reality Judgment and Presence Questionnaire (RJPQ) and ITC-Sense of Presence Inventory (ITC-SOPI). The results showed that the VRE induced a sense of presence and was felt as real for both groups, without differences in the experience of "ease" with the VRE, sense of physical space, or the ecological validity assigned to the virtual kitchen and eating virtually. However, the ED patients reported paying more attention and experiencing greater emotional involvement and dysphoria after virtual eating. The results suggest that the VRE was clinically meaningful to the ED patients and might be a relevant therapy tool for normalizing their eating patterns.
\end{abstract}

(C) 2013 Elsevier Inc. All rights reserved.

\section{Introduction}

\subsection{Eating Disorders and disordered eating}

Eating Disorders (ED) are characterized by altered eating patterns and the overvaluation of figure and weight in the self-concept. Among them, the DSM-IV-TR [1] includes Anorexia Nervosa, Bulimia Nervosa, and ED-not otherwise specified. The latter is especially important given that approximately $60 \%$ of ED outpatient cases are diagnosed in this way [2-4], with the binge-eating disorder being especially relevant considering its prevalence and relationship with obesity [5]. The course and outcome of ED can vary considerably. In some cases, the disorder only develops partially, while in others it becomes chronic and is

* Corresponding author. Department of Personality, Assessment and Psychological Treatment, Faculty of Psychology, Avda. Blasco Ibáñez, 21, 46010-Valencia. Spain. Tel.: +34 963983494; fax: +34 963864669.

E-mail address: perpinya@uv.es (C. Perpiñá). maintained for years. Moreover, it has become increasingly important to consider ED and obesity as two poles on the same continuum of problems related to eating and weight. These two conditions include a wide variety of types of disordered eating as, without meeting all the criteria, they represent anomalous functioning patterns, including body dissatisfaction, unhealthy behaviors to lose weight, thinness or overweight, with alarming epidemiological figures. Screening studies in the USA have estimated that $25 \%$ of girls and $11 \%$ of boys present a disordered eating pattern with a degree of severity requiring a posterior clinical assessment [6].

The most widely-accepted form of psychotherapy for treating ED, mainly bulimia nervosa and binge eating disorder, is cognitive behavior therapy (CBT). CBT trains patients to change their patterns of thinking and action to prepare them to face their fears [7]. However, despite the progress made, the severity, chronicity and complications with obesity problems observed in these disorders demonstrate a lack of treatments completely capable of addressing 
their complexity $[5,8]$. Currently available treatments have the main objective of stabilizing eating patterns in order to achieve weight regulation, but these therapeutic strategies must be optimized [9].

\subsection{Virtual Reality as a clinical tool}

Currently, a great expansion is occurring in computational technologies and, specifically, Virtual Reality (VR) technologies, in many healthcare areas. VR techniques have been shown to be effective as therapeutic tools in treating various psychological disorders and problems, changes in habits, and rehabilitation, with noteworthy success [10-13]. In the last 15 years, interest in exposure techniques designed and developed in VR has increased; therefore, clinicians have created virtual environments that allow patients to confront their fears [14]. Unlike exposure using the imagination, patients are engaged by several of their senses, heightening the realism of the context. Unlike exposure "in vivo", patients are in a safe, confidential place with the therapist in complete control [15]. Finally, it is much more difficult to control the variables in real-life situations than in VR environments.

VR was first used in the ED field in the treatment of body image alterations in a group of subclinical participants worried about food and physical appearance [16]. Later, a controlled study developed a CBT treatment supported by VR techniques for body image disturbances, and applied it to a sample of ED patients [1-18]. Results showed that patients treated in the CBT plus VR condition showed significantly greater improvement, not only in body image, but also on psychopathologyrelated indices, with further improvements after one year.

The use of VR has also extended to other alterations in eating behavior, obtaining similar results when applying cognitive therapy combined with VR in treating body image disturbances in patients with binge-eating disorder and obesity [18-20].

Another necessary treatment element, both in ED and obesity, is eating pattern normalization. Patients with ED show anxiety when eating high-calorie food and in situations where their body is displayed or they come into contact with other people [21]. These cues related to food aversion (specifically high-calorie food) are included in most ED assessment instruments and therapy programs [22].

Considering the advantages and challenges VR presents as a therapeutic tool, it is logical to propose that VR can help to deal with aspects of eating that are difficult to include in therapeutic sessions, such as exposure to food or eating certain "forbidden" foods by the patients [23]. In recent years, studies have shown that exposure to virtual food produces the same sensations as exposure to real food, using both non-immersive [24] and immersive environments [25].

\subsection{Current study}

Until now, VR applications in disordered eating behavior have basically focused on body image intervention, addres- sing the topic of eating and disordered eating habits tangentially. However, this technology may help to improve aspects of the behavioral-cognitive strategies in the normalization of eating and regulating the urge to eat.

The main purpose of this study was to carry out the clinical validation of VR software about environments involving food and eating for use in normalizing the eating behavior of ED patients and people with disordered eating. To this aim, differences in the VR experiences (in this case, non-immersive) of ED patients and non-clinical individuals were analyzed. We hypothesize that ED patients experience VR environments related to food and eating with more discomfort, fear and avoidance than non clinical participants, since these elements are emotionally and clinically significant to the patients' problem.

\section{Method}

\subsection{Participants}

The Clinical Group (CG) consisted of 22 females with an ED diagnosis [11 Anorexia nervosa (AN), 4 Bulimia nervosa (BN), 7 Not otherwise specified ED (EDNOS)], according to DSM-IV-TR criteria (APA, 2002), recruited from the psychiatric Departments of Dr. Peset Hospital and Bellvitge University Hospital (Spain). The mean age of the clinical group was 24.50 years $(\mathrm{SD}=7.92)$, with a mean Body Mass Index (BMI) of 19.93 ( $\mathrm{SD}=4.13$; range: $16.03-29.35)$. The majority were single $(68.2 \%)$ with a medium socioeconomic level (68.2\%) and secondary studies (77.3\%).

The Healthy Comparison (HC) group consisted of 37 female psychology students in their last year at the University of Valencia. The mean age of this group was 24.24 years $(\mathrm{SD}=6.53)$, with $\mathrm{BMI}=21.64 \quad(\mathrm{SD}=3.02$; range: $18.12-27.73)$. The majority were single $(86.5 \%)$ with a medium socio-economic level (78.4\%).

\subsection{Software and hardware}

The virtual environment consisted of a kitchen with two main areas. The first is the prep area, with a countertop, cabinets, burners and a fridge that contains all of the elements necessary to prepare a meal, serve it and then eat it "virtually." When the food is eaten, it is accompanied by the sound of chewing and the portions of food on screen gradually disappear. The foods are located in the fridge, cabinets and shelves, which the user can access freely or block if desired. The second kitchen area is mainly for eating foods the right way: there are a table and a chair for the patient to sit along with all the necessary items for a meal (dishes, a glass, silverware, a napkin). The eating style can be set to fast or slow. The size of certain foods can be increased or decreased (chocolate, potatoes, pizza, etc.) so that they take up the whole screen (i.e. the kitchen) or get so small that they disappear. There is also an option for alternative behaviors (making a phone call or turning on the radio) in 
order for the patient to distract him/herself from the impulse to eat. In addition, the software allows therapists to help their patients to address negative thoughts, think positive thoughts and training in positive self-instruction. Finally, the software provides visuals of the positive or negative consequences of the decision and behavior that the patient has just displayed (an image of body organs depicted as happy or sad). However in order to get homogenous conditions in all the participants, the assessment in this study was only carried out in a significant virtual action: "eat virtually a forbidden food", usually a pizza. See Fig. 1.

This non-immersive virtual environment was displayed on a 3-GHz Pentium V laptop with 1 GB RAM, a $256 \mathrm{Mb}$ graphic card, a 19-in. monitor and a 2D mouse. The participants were comfortably seated in front of the computer watching the monitor-screen located at a distance of $50 \mathrm{~cm}$, next to the clinician, and with the lights in the room turned off.
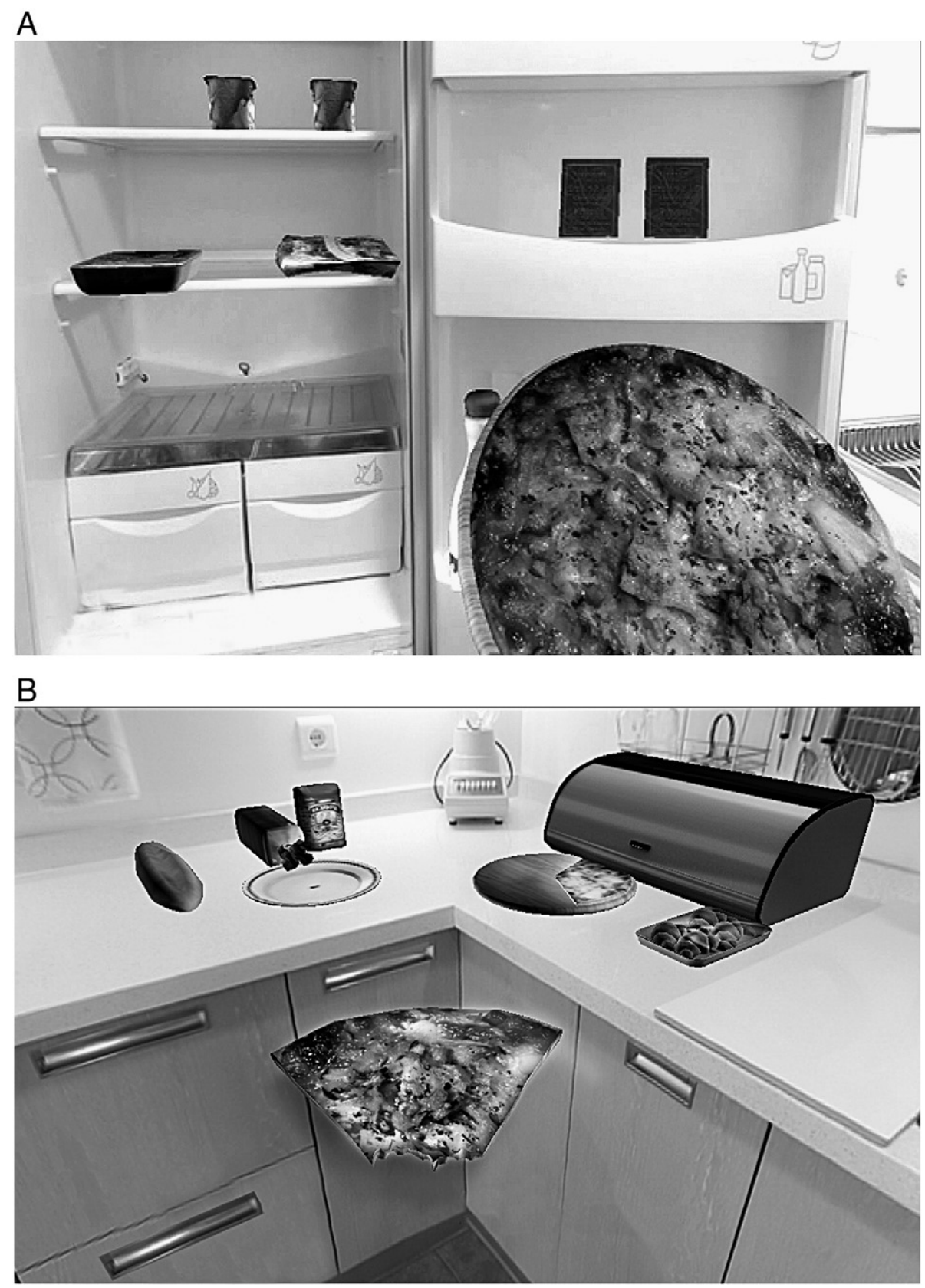

Fig. 1. Pictures of the virtual environment. A: Close-up of the pizza. B: Partially eaten pizza. There are two main zones. The first is a work area, which includes a counter, cupboards, stove and fridge, where the patients find the necessary virtual elements to prepare food, serve it and eat it if desired. The food is located in the fridge, cupboard and shelves. The second area is devoted mainly to eating correctly: sitting at a table with all the necessary elements, plates, a glass, silverware and napkin. The eating style can be controlled to make it faster or slower by the clinician. This is accompanied by the sound of chewing, the progressive disappearance of food portions and the progressive view of leftovers and extra juices as they are disappearing from the plate. 


\subsection{Measures}

\subsubsection{Clinical measures}

Beck Depression Inventory (BDI-II) [26]. This 21-item self-report measures depressive symptomatology. The individual has to choose one out of the four statements that best represents his/her feelings in the last two weeks. The Spanish version [27] was administered (Cronbach's $\alpha$ : 0.85).

Beck Anxiety Inventory (BAI) [28]. This 21-item questionnaire measures anxiety severity in an adult population on a four-point scale. The Spanish validation [29] was administered (Cronbach's $\alpha$ : 0.95).

The Restraint Scale (RS) [30]. This self-report assesses eating behavior patterns characterized by alternating strict diets with episodes of loss of control with food. It consists of 10 items responded to using a four- or five-point scale. Its internal consistency exceeds 0.75 . The cut-off score is 16 . This scale was only administered to the HC Group for screening purposes.

\subsubsection{Measures of the virtual experience}

To assess the reality attribution and sense of presence produced by the virtual environment, three instruments were used:

Clinically significant measures related to the virtual experience. Seven questions with a Likert format (0: not at all-10: absolutely) were designed specifically for this study. During the virtual experience, participants were asked to assess its clinical relevance and degree of reality. Before eating a forbidden food (a piece of pizza) "virtually", they were asked about their desire to eat, control of the urge to eat, fear, and avoidance of eating. After eating the virtual pizza, they were asked about the feeling of putting on weight, the degree of similarity to a real experience, the reality of the virtual experience, what emotion they felt and what activity they preferred to do after eating.

The Reality Judgment and Presence Questionnaire $(R J P Q)$ [31] is a post-test subjective measure assessing presence and reality judgment. It consists of 56 self-report items (Likert scale format ranging from 0: not at all to 10: absolutely) with 6 factors: Emotional involvement; Reality Judgment and Presence; Interaction and external correspondence; Influence of the Quality of the Software; Software Easiness; Satisfaction with the experience; and Attention (Cronbach's $\alpha$ : 0.82).

The revised version of the ITC-Sense of Presence Inventory (ITC-SOPI) [32]. This 44-item post-test self-report has a five-point Likert scale (1: strongly disagree-5: strongly agree) and four factors: Sense of physical space; Engagement; Ecological Validity; and Negative Effects (Cronbach's $\alpha: 0.94-0.76)$.

\subsection{Procedure}

Patients were interviewed face-to-face and diagnosed using the Structured Clinical Interview (SCID-I) for DSMIV [33]. The comparison group of healthy eating participants was recruited in final-year Psychology courses at the University of Valencia (Spain), and received partial course credit for their collaboration. Only students who reported no psychological disorders or psychological or psychiatric treatments in the last year were included in the study.

Prior to the study, individuals received a brief explanation and signed an informed consent. Before the virtual experience, participants completed the BDI, BAI, and RS. After the virtual experience (lasting 20-25 min, including the 7 questions answered during the experiences), the RJPQITC-SOPI were administered; finally, mainly the $\mathrm{HC}$ participants were weighed and measured to calculate their BMI. This study received the approval of Dr. Peset Hospital, Bellvitge University Hospital and the University of Valencia Ethics Committees.

\subsection{Statistical analyses}

Analyses were performed with SPSS v.19. To analyze differences between groups on the relevant variables, $t$ tests with Levene's corrections were used, and $\chi^{2}$ when nominal variables were compared. Effect sizes were calculated with Cohen's $d$. A $d$ value $<0.20$ was considered a small effect size, and $>0.8$ was considered large [34].

\section{Results}

\subsection{Group differences in socio-demographic and clinical variables}

Differences in socio-demographic variables were calculated between patients and comparison group. Both groups were equally distributed on BMI $\left(t_{(57)}=1.83, p=.07\right)$, age $\left(t_{(37.8)}=-0.13, p=.90\right)$ and socio-economic level $\left(\chi_{3}^{2}=\right.$ $2.08, \mathrm{p}=.55)$. However, differences were found in marital status $\left(\chi_{3}^{2}=11.58, p=.009\right)$ and level of studies $\left(\chi_{2}^{2}=\right.$ 47.13, $\mathrm{p}=.001)$.

Regarding clinical measures, patients scored higher than the comparison group on depression (BDI; patients: $\mathrm{M}=$ 29.28; $\mathrm{SD}=14.24$; HC group: $M=4.72 ; S D=4.60 ; t_{(22.46)}=$ 7.67; $p .0001$ ) and anxiety measures (BAI; patients: $\mathrm{M}=$ 23.24; $\mathrm{SD}=14.58$; HC group: $M=7.61 ; S D=5.22 ; t_{(23.02)}=$ $4.73 ; p<.0001)$. As expected, the HC group showed low scores on the RS $(M=8.57 ; S D=4.32)$, indicating that they were not a "subclinical" ED sample.

\subsection{Virtual experience measures}

When ED patients were told to eat "virtual-forbiddenfood" (pizza), before eating they showed moderate-high scores (around 7) on Control urge to eat, Fear, and Avoidance. However, their desire to eat was low. After eating, they reported a feeling of putting on weight. Additionally, patients showed a high reality judgment and sense of presence (see Table 1). In contrast, when healthy participants were told to eat the "virtual pizza", they reported a high Desire to eat and Control urge to eat, but low Fear, 
Table 1

Descriptive data and $t$ test of virtual experience measures for patient and control groups.

\begin{tabular}{|c|c|c|c|c|c|}
\hline Measures of the Virtual experience & $\begin{array}{l}\text { Clinical Group } \\
(\mathrm{CG})\end{array}$ & $\begin{array}{l}\text { Healthy Comparison } \\
\text { (HC) }\end{array}$ & $t$ & $d f$ & $d$ \\
\hline \multicolumn{6}{|c|}{ Relevant clinical measures-During exposure } \\
\hline Desire to eat & $4.32(3.93)$ & $7.56(2.06)$ & $3.59^{* *}$ & 27.99 & 0.97 \\
\hline Control of urge to eat & $7.05(3.00)$ & $8.30(3.10)$ & 1.49 & 57 & 0.40 \\
\hline Fear & $7.36(2.90)$ & $0.53(1.53)$ & $10.23 * * *$ & 28.03 & 2.75 \\
\hline Avoidance & $7.68(3.09)$ & $1.35(2.86)$ & $7.97 * * *$ & 57 & 2.14 \\
\hline Feeling of Putting on Weight & $6.67(3.07)$ & $0.38(1.30)$ & $8.94 * * *$ & 24.12 & 2.41 \\
\hline Similarity with the real experience & $6.38(2.84)$ & $6.08(1.82)$ & 0.44 & 29.50 & 0.12 \\
\hline Reality of the experience & $7.10(2.53)$ & $5.95(1.58)$ & $2.12 *$ & 55 & 0.57 \\
\hline \multicolumn{6}{|c|}{ Reality and presence measures-Post exposure } \\
\hline RJPQ-Emotional involvement & $5.72(2.64)$ & $4.38(2.03)$ & $2.20^{*}$ & 57 & 0.59 \\
\hline RJPQ-Reality Judgment and Presence & $6.77(1.86)$ & $6.28(1.87)$ & 0.98 & 57 & 0.26 \\
\hline RJPQ-Interaction, & $7.02(1.68)$ & $7.16(1.36)$ & 0.35 & 57 & 0.09 \\
\hline RJPQ-Quality & $6.01(2.59)$ & $7.25(1.63)$ & 2.01 & 31.05 & 0.54 \\
\hline RJPQ-Easiness & $6.86(2.08)$ & $7.76(1.56)$ & 1.87 & 57 & 0.50 \\
\hline RJPQ-Satisfaction & $6.62(2.33)$ & $7.05(1.86)$ & 0.78 & 57 & 0.21 \\
\hline RJPQ-Attention & $5.30(2.16)$ & $3.92(1.75)$ & $2.67 * *$ & 57 & 0.72 \\
\hline ITC-SOPI-Sense of physical space & $3.35(0.68)$ & $3.09(0.53)$ & 1.62 & 57 & 0.44 \\
\hline ITC-SOPI-Engagement & $3.16(0.70)$ & $3.06(0.65)$ & 0.54 & 57 & 0.14 \\
\hline ITC-SOPI-Ecological Validity & $3.59(0.84)$ & $3.60(0.64)$ & 0.04 & 26.83 & 0.01 \\
\hline ITC-SOPI-Negative Effects & $1.73(0.67)$ & $1.26(0.31)$ & $3.12 * *$ & 57 & 0.84 \\
\hline
\end{tabular}

RJPQ: Reality Judgment and Presence Questionnaire; ITC-SOPI: ITC-Sense of Presence Inventory. Data are expressed as Mean (Standard deviation). $d f$ : degrees of freedom; $d$ : Cohen's d.

$$
\begin{aligned}
& * p<.05 . \\
& * * p<.01 . \\
& * * * p<.001 .
\end{aligned}
$$

Avoidance, and Feeling of putting on weight (scores close to 0 ), with noteworthy effect sizes for the differences. Regarding the "reality" of the experience, the control group showed moderate scores (around 6). There were no significant differences between groups, only on Control urge to eat and Similarity to the real experience (see Table 1).

After eating, participants were asked about their feelings and urges. Five patients $(22.7 \%)$ felt the urge to continue eating, 4 felt upset (18.2\%), 4 felt anxious (18.2\%), 3 felt guilty $(13.6 \%), 2$ felt the urge to purge (9\%), 2 felt good $(9 \%)$, one patient felt bloated $(4.5 \%)$, and one patient answered "others" (4.5\%). In contrast, 30 of the $37 \mathrm{HC}$ participants $(81.1 \%)$ felt good after eating the virtual food, 4 felt the urge to continue eating $(10.8 \%), 2$ felt bloated (5.4\%), and one (2.7\%) answered "other". Next, participants were asked what they would like to do after eating. Seven patients $(31.8 \%)$ wanted to do exercise to "compensate" for the previous eating, 6 patients $(27.3 \%)$ wanted to continue eating, another $6(27.3 \%)$ wanted to continue with their daily routine, 2 wanted to purge $(9.1 \%)$, and one chose "others". In contrast, $30 \mathrm{HC}$ participants $(81.1 \%)$ wanted to continue their daily routine, 5 preferred to continue eating the virtual food (13.5\%), and 2 wanted to compensate for the food eaten by doing exercise $(5.4 \%)$.

Considering the virtual environment as a global experience, in general, all participants showed satisfactory results regarding the sense of presence and reality judgment, with scores around 7 on the $R J P Q$ and around 4 on the ITC-SOPI.
Curiously, for the HC group, emotional involvement and attention were especially low. Moreover, in both groups the level of negative effects of the virtual experience was low. Means and standard deviations on both questionnaires are shown on Table 1. A comparison of the CG and HC groups showed no differences in the Reality Judgment and Presence, Interaction, Quality, Easiness, Satisfaction, Sense of physical space, Engagement and Ecological validity of the virtual environment. However, the CG group scored higher on Emotional Involvement, Attention and Negative Effects.

\section{Discussion}

The treatment of choice for most ED is CBT, which usually begins with the normalization of eating patterns. However ED patients feel great fear and anxiety when exposed to food, and especially "forbidden" food (e.g. highcaloric). The aim of this study was to validate the clinical meaning of a non-immersive virtual environment designed for use in normalizing the eating pattern in ED patients and people with disordered eating. The results showed that the virtual environment induced a sense of presence and felt real in both groups, with no differences in experiencing "easiness", satisfaction with the environment, sense of physical space or the ecological validity assigned to the virtual kitchen and to the actions carried out in it, in this case, 
virtually eating a piece of pizza. However, the ED patients reported paying more attention to the virtual environment, and they experienced greater emotional involvement.

When the analyses of the virtual experience focused on elements relevant to the clinical eating problems (eating a pizza), the VR software was not only able to produce the same emotions as in real life, but most importantly, it also produced significant differences between groups, with the ED patients reporting more fear, avoidance and feelings of putting on weight related to eating the virtual pizza, and with a large effect size for this difference. VR has been shown to arouse similar responses in participants to those elicited by the real situation [35]. Several studies [36,37] support the idea that the emotions (such as fear or anxiety) felt during the virtual experience are related to the sense of presence, defined as the feeling of "being in" the virtual environment [38]. Studies indicate that in environments capable of arousing emotions, whether in non clinical participants [39] or clinical ones [40], the level of emotional reactivity during the virtual experience increased the sense of presence proportionally. As suggested by other authors [41], before using any VR environment in psychopathology, researchers have to be sure that the virtual environment elicits roughly the same degree of emotion as the real-life situation.

Other studies have assessed the ability of VR to induce emotional, cognitive, and behavioral responses in ED patients, similar to those observed in real life. A pilot study was conducted with a small sample of ED patients to analyze the use of VR to assess and treat binge-eating episodes [42]. The results showed that the virtual environment was able to provoke the undesirable features present in binge-eating episodes, such as anxiety, impulse to over-eat, and guilt feelings. Ferrer-García et al. [24] found that, compared with controls, patients with ED showed significantly higher levels of anxiety and depressed mood in virtual environments that were meaningful and relevant to their disorder, such as ones where they had eaten a virtual food or visited the swimming pool. Another study comparing emotional reactions to real food, VR food, and photographs of food in ED patients and healthy control participants found that real and VR food produced comparable emotional reactions in ED patients, with this reaction being even stronger than the one produced by photographs of food [25]. The results obtained with both in vivo and VR exposure appear to be similar, which indicates that these environments could be used for both evaluative and therapeutic aims.

The virtual environment used in the present study was non-immersive. As indicated in the results of the majority of studies with clinical and subclinical participants, the feeling of presence and the attribution of reality reported by the participants are largely due to the relevance and clinical significance of the virtual environment in their disorder. A relevant virtual environment design for the specific disorder, selecting key elements and actions for the problem, is what makes the environment emotionally significant for them [43].
The virtual environment in the present study was recently described as a therapeutic tool, as a component of virtual exposure to food, in two case reports, one about a patient with AN restrictive subtype [44] and another about a patient with AN purgative subtype [45]. After virtual exposure to food and eating "virtually", the patients showed less anxiety and fear related to food, while the eating symptomatology diminished and their BMI increased.

The essential issue in using VR in treating mental disorders is to design environments that are significant to the patient's psychopathology, even when the element to be re-created is something as 'basic' as food. Thus, the usefulness of VR does not lie in 're-creating' reality, but rather in helping patients to face their mental representations and fears. Using VR techniques in the CBT for ED may be useful for motivation to change, feelings of self-efficacy, elimination of binges, control over emotional eating, and adherence to treatment.

The present study has several limitations. Participants' responses are based on self-report instruments and subjective experiences that may introduce a source of bias in the results. Complementing the procedure used, the assessment could be improved by including physiological indices (e.g. heart rate, pulse, galvanic skin reactivity). Another limitation is the lack of sample power, justified by the exploratory nature of this pilot study. The scarce number of clinical participants makes difficult a differential analysis by diagnostic subgroups (e.g. evaluating separately restricting $\mathrm{AN}$, binge-purging $\mathrm{AN}$, normal weight BN, obese BED). Future studies should include different ED subtypes in broader samples in order to analyze the different emotional reactions and eating patterns in relation to the VE. Since VR seems capable of producing anxiety and food avoidance and awakening the urge to eat, opportunities arise to design components for preventing and managing binging and emotional eating in ED patients.

Summarizing, these results suggest that this VR software was clinically meaningful to the ED patients and might be a relevant therapeutic complement to traditional therapy.

\section{Acknowledgments}

This study was partially supported by Ministerio de Sanidad, Spain (FIS-PI051937) and Consellería de Sanidad, Valencia, Spain (SMI 3/2008) granted to C. Perpiñá.

Centro de Investigación Biomédica en Red Fisiopatología de la Obesidad y Nutrición is an initiative of ISCIII.

\section{References}

[1] American Psychiatric Association. DSM-IV-TR: diagnostic and statistical manual of mental disorders. 4th ed. revised. Washington, DC: American Psychiatric Association; 2000.

[2] Machado PP, Machado BC, Goncalves S, Hoek HW. The prevalence of eating disorders not otherwise specified. Int $\mathrm{J}$ Eat Disorder 2007;40:212-7.

[3] Dunn EC, Geller J, Brown KE, Bates ME. Addressing the EDNOS issue and improving upon the utility of DSM-IV: classifying eating 
disorders using symptom profiles. Eur Eat Disord Rev 2010;18: 271-80.

[4] Helverskov JL, Lyng B, Clausen L, et al. Empirical support for a reclassification of eating disorders NOS. Eur Eat Disord Rev 2011;19:303-15.

[5] Villarejo C, Fernández-Aranda F, Jiménez-Murcia S, et al. Lifetime obesity in patients with eating disorders: increasing prevalence, clinical and personality correlates. Eur Eat Disord Rev 2012; 20:250-4.

[6] Austin SB, Ziyadeh NJ, Forman S, Prokop LA, Jacobs D. Screening high school students for eating disorders. Reports of a national initiative. Prev Chron Disord 2008;5:1-10.

[7] National Institute of Mental Health. Eating disorders [brochure]. No. 11-4901. Available at: NIH Publication 2011 www.nimh. nih.gov/health/publications/eating-disorders/eating-disorders.pdf. www.nlm.nih. gov/medlineplus/spanish/.

[8] Wilson GT. Eating disorders, obesity and addiction. Eur Eat Disord Rev 2010;18:341-51.

[9] Hart S, Abraham S, Franklin R, Russell J. Weight changes during inpatient refeeding of underweight eating disorder patients. Eur Eat Disord Rev 2011;19:390-7.

[10] Botella C, Villa H, Garcia-Palacios A, Baños RM, Perpiñá C, Alcañiz M. Clinically significant virtual environments for the treatment of panic disorder and agoraphobia [electronic version]. Cyberpsychol Behav 2004;7:527-35.

[11] Krijn M, Emmelkamp PMG, Olafsson RP, Biemond R. Virtual reality exposure therapy of anxiety disorders: a review. Clin Psychol Rev 2004;24:259-81.

[12] Powers MB, Emmelkamp PMG. Virtual reality exposure therapy for anxiety disorders. J Anxiety Disord 2008;22:561-9.

[13] Carrard I, Fernandez-Aranda F, Lam T, et al. Evaluation of a guided internet self-treatment programme for bulimia nervosa in several European countries. Eur Eat Disord Rev 2011;19:138-49.

[14] Bush J. Viability of virtual reality exposure therapy as a treatment alternative. Comput Hum Behav 2008;24:1032-40.

[15] Wiederhold BK, Jang DP, Gevirtz RG, Kim SI, Kim IY, Wiederhold MD. The treatment of fear of flying: a controlled study of imaginal and virtual reality graded exposure therapy [electronic version]. IEEE T Inf Technol B 2002;6:218-23.

[16] Riva G, Melis I, Bolzoni M. Treating body-image disturbances. Commun ACM 1997;40:69-71.

[17] Perpiñá C, Botella $C$, Baños RM, et al. Body image and virtual reality in eating disorders: exposure by virtual reality is more effective than the classical body image treatment? Cyberpsychol Behav 1999;2:149-59.

[18] Perpiñá C, Marco JH, Botella $\mathrm{C}$, et al. Tratamiento de la imagen corporal en los trastornos alimentarios mediante tratamiento cognitivo-comportamental apoyado con realidad virtual: resultados al año de seguimiento. Psicol conduct 2004;12:519-37.

[19] Riva G, Bacchetta M, Baruffi M, Rinaldi S, Vincelli F, Molinari E. Virtual reality-based experiential cognitive treatment of obesity and binge-eating disorders. Clin Psychol Psychoth 2000;7:209-19.

[20] Riva G, Bacchetta M, Cesa G, Conti S, Molinari E. The use of VR in the treatment of eating disorders. Stud health technol inform 2004;99:121-63.

[21] Pallister E, Waller G. Anxiety in the eating disorders: understanding the overlap. Clin Psychol Rev 2008;28:366-86.

[22] Overduin J, Jansen A. Food cue reactivity in fasting and non-fasting subjects. Eur Eat Disord Rev 1996;4:249-59.

[23] Lock L, Williams H, Bamford B, Lacey JH. The St George's Eating Disorders Service Meal Preparation Group for Inpatients and Day Patients Pursuing Full Recovery: a pilot study. Eur Eat Disord Rev 2012;20:218-24.

[24] Ferrer-García M, Gutiérrez-Maldonado J, Caqueo-Urízar A, Moreno E. The validity of virtual environments for eliciting emotional responses in patients with eating disorders and in controls. Behav Modif 2009;3:830-54.

[25] Gorini A, Griez E, Petrova A, Riva G. Assessment of the emotional responses produced by exposure to real food, virtual food and photographs of food in patients affected by eating disorders. Ann Gen Psychiatr 2010;9:1-30.

[26] Beck AT, Steer RA, Brown GK. Manual for the Beck Depression Inventory-II. San Antonio (Tex): The Psychological Corporation; 1993.

[27] Sanz J, Perdigón AL, Vázquez C. Adaptación Española del Inventario para la Depresión de Beck II (BDI-II): 2. Propiedades psicométricas en población general. Clínica y Salud 2003;14:249-80.

[28] Beck AT, Steer RA. Beck Anxiety Inventory manual. San Antonio: Harcourt Brace and Company; 1993.

[29] Comeche MI, Díaz MI, Vallejo MA. Cuestionarios, Inventarios y Escalas. Ansiedad, Depresión y Habilidades Sociales. Madrid: UNED, Fundación Universidad-Empresa; 1995.

[30] Herman CP, Polivy J. Anxiety, restraint and eating behavior. J Abnorm Psychol 1975;84:66-72.

[31] Baños RM, Botella C, García-Palacios A, Villa H, Perpiñá C, Alcañiz $\mathrm{M}$. Presence and reality judgment in virtual environments: a unitary construct? Cyberpsychol Behav 2000;3:327-35.

[32] Lessiter J, Freeman J, Keogh E, Davidoff J. A cross-media presence questionnaire: the ITC-Sense of Presence Inventory. Presence-Teleop Virt 2001;10:282-98.

[33] First M, Gibbon M, Spitzer R, Williams J. Users guide for the Structured Clinical Interview for DSM IV Axis I Disorders Research Version (SCID-I, version 2.0). New York: New York State Psychiatric Institute; 1996.

[34] Cohen J. Statistical power analysis for the behavioral sciences. Hillsdale (N.J.): Lawrence Earlbaum Associates; 1988.

[35] Alsina-Jurnet I, Carvallo-Beciu C, Gutiérrez-Maldonado J. Validity of virtual reality as a method of exposure in the treatment of test anxiety. Behav Res Meth 2007;39:844-51.

[36] Bouchard S, St-Jaques J, Robillard G, Renaud P. Anxiety increases the feeling of presence in virtual reality. Presence-Teleop Virt 2008;17:376-91.

[37] Juan MC, Pérez D. Using augmented and virtual reality for the development of acrophobic scenarios. Comparison of the levels of presence and anxiety. Comput \& Graph 2010;34:756-66.

[38] Witmer B, Singer M. Measuring presence in virtual environments: a presence questionnaire. Presence-Teleop Virt 1998;7:225-40.

[39] Regenbrecht HT, Schubert TW, Friedmann F. Measuring the sense of presence and its relations to fear of heights in virtual environments. Int J Hum-Comput Int 1998;10:233-49.

[40] Schuemie MJ, Bruynzeel M, Drost L, Brinckman M, de Haan G, EmmelKamp PMG. Treatment of acrophobia in virtual reality: a pilot study. In: Broeckx F, \& Pauwels L, editors. Proceedings of Euromedia; 2000. p. 271-5.

[41] Slater M, Pertaub DP, Barker C, Clark DM. An experimental study on fear of public speaking using a virtual environment. Cyberpsychol Behav 2006;9:627-33.

[42] Perpiñá C, Botella C, Quero S, Marco JH, Baños RM. Design of clinically significant virtual-environments for binge eating episodes. Presented at the VII European Council on Eating Disorders, ECED; 2001. Barcelona, Spain.

[43] Perpiñá C, Botella C, Baños RM. Virtual reality in eating disorders. Eur Eat Disord Rev 2003;11:261-78.

[44] Cardi V, Krug I, Perpiñá C, Mataix D, Roncero M, Treasure J. The use of a non immersive virtual reality program in anorexia nervosa: a single case report. Eur Eat Disord Rev 2012;20:240-5.

[45] Perpiñá C, Ferrero A, Carrió C, Roncero M. Normalization of the eating pattern in a patient with anorexia nervosa, purgative subtype, with the support of a virtual environment. A case study. Ann Clin Health Psychol 2012;8:7-21. 